\title{
Análisis de propiedades del transporte de humedad en bloques de hormigón
}

\author{
Analysis of properties of the transport of moisture \\ in concrete blocks
}

N. L. Mustelier $^{(*)}$, J. C. Rocha ${ }^{(* *)}$, M. Cheriaf ${ }^{(* *}$

\section{RESUMEN}

Las propiedades de absorción de humedad de los bloques de hormigón tienen influencias significativas en el comportamiento a la penetración de agua de lluvia. El conocimiento de estas propiedades es necesario para poder contar con información técnica que permita la construcción de paredes con vida útil prolongada.

En el presente articulo se realizó un análisis comparativo de dos bloques de hormigón estructurales en relación a sus propiedades físicas y de transporte de humedad: porosidad abierta, masa especifica así como la tasa inicial de absorción (IRA), absorción total y la absorción en función del tiempo, con la obtención de la sorptividad como código de medición preciso y confiable.

Los resultados mostraron que la sorptividad no es aplicable en el caso de los bloques de hormigón $\mathrm{D}_{2}$ y se presenta la curva media de absorción en función del tiempo como un nuevo parámetro de caracterización que permite analizar el comportamiento y las peculiaridades de los bloques desde el instante inicial hasta la saturación.

$686-4$

Palabras clave: Bloques de hormigón; tasa inicial de absorción; sorptividad.

\section{SUMMARY}

The moisture absorption properties in the concrete blocks have a significant influence on the performance of the penetration of rainwater. Knowledge of these properties is necessary to have technical information that allows the construction of walls with long life.

In this paper we conducted a comparative analysis of two structural concrete blocks in relation to their physical properties and moisture transport: open porosity, specific mass and the initial rate of absorption (IRA), the total absorption and uptake versus time, obtaining the sorptivity as code for measuring the absorption accurate and reliable.

The results obtained show that the sorptivity in the case of concrete block $D_{2}$ is not applicable and presents the absorption average curve versus time as a characterization parameter with great potential, once can be observed the behavior of the blocks from the initial time to saturation, showing the characteristics between the different block types analyzed.

Keywords: Concrete blocks; initial rate of absorption (IRA); sorptivity.

\footnotetext{
(*) Universidade do Porto, (Portugal).

(**) Universidade Federal de Santa Catarina, Florianópolis (Brasil).

Persona de contacto/Corresponding author: niubis@yahoo.com (N. Mustelier)
} 


\section{INTRODUCCIÓN}

El estudio de las propiedades de transporte de humedad de los elementos constructivos de una pared se realiza generalmente como predicción de su comportamiento, código de buenas prácticas o para la construcción de edificios con vida útil prolongada (1). Las unidades de una mampostería (bloques de hormigón) pueden ser caracterizadas en relación a su porosidad, distribución de poros, textura superficial, así como por su capacidad de absorción de agua. En diversos estudios se aborda que la tasa inicial de absorción (IRA) de elementos de construcción ejerce un importante papel en la resistencia a la penetración del agua (2) (3).

Otras investigaciones afirman que la tasa inicial de absorción no representa con fidelidad el comportamiento absorbente de un elemento de construcción porque se considera que en un minuto no es posible medir la capacidad de absorción de agua y que en la práctica pueden obtenerse resultados superiores de la tasa inicial de absorción, ya que las fuerzas capilares continúan ejerciendo su función durante un período prolongado de tiempo (4). Diversas investigaciones obtuvieron resultados que demuestran una variabilidad considerable debido a la simplicidad de este ensayo (5).

La tasa inicial de absorción no permite definir las características de absorción, solamente indica la capacidad de succión de agua del mortero por el elemento de construcción, enfatizándose la necesidad de un análisis para complementar con otras propiedades, como la porosidad del material, y así poder tener una caracterización global de los elementos de construcción. En la literatura, numerosos trabajos (6) (7) (8) se refieren a la absorción de agua de diferentes materiales porosos así como a la ley de absorción de líquido dependiente del tiempo, a través de la forma general [1]:

$$
i=S \cdot t^{1 / 2}
$$

Esta formula representa la cinética básica del proceso de succión, donde el agua absorbida por un material poroso por unidad de superficie ( $m$ ) aumenta en la medida que la raíz cuadrada del tiempo $(t)$ transcurre, definiéndose $(S)$ como el coeficiente capilar. Este se obtiene, antes que ocurra la saturación del material (9).

Teniendo en cuenta, la absorción unidimensional del agua, la curva resultante del volumen de agua absorbido por área de superficie frente a la raíz cuadrada del tiempo de absorción debe ser proporcional. La pendiente de la curva donde la parte es lineal, suministra los datos para calcular este coeficiente capilar y se define como la sorptividad (S) del material (10) (11). La teoría de la sorptividad se presenta como una propuesta para la realización de mediciones confiables de las propiedades de absorción de la superficie de los diferentes materiales porosos, pero existen limitaciones en la aplicación de esta teoría para los elementos de construcción.

En esta investigación se abordó la caracterización de dos tipos de bloques de hormigón en relación a las propiedades físicas y de transporte de humedad: porosidad abierta, masa específica, absorción total, tasa inicial de absorción y absorción en función del tiempo. Se obtuvo la sorptividad (7 horas) con el propósito de validar su aplicación para el caso de bloques de hormigón estructurales así como se presentó la curva media de absorción como un nuevo parámetro de caracterización que permite analizar el comportamiento y las peculiaridades de los bloques desde el instante inicial hasta la saturación.

\section{PROCESO EXPERIMENTAL}

Se analizaron dos tipos de bloques de hormigón estructurales ahuecados, con dimensiones de $39 \mathrm{~cm} \times 19 \mathrm{~cm} \times 14 \mathrm{~cm}$. Los bloques fueron producidos a escala industrial y vibro-prensados para obtener bloques con dos densidades. Estos fueron designados como serie $\mathrm{D}_{1}$ para el caso de los bloques más densos y $\mathrm{D}_{2}$ para la serie de bloques menos densos.

El programa experimental incluyó tres pruebas diferentes para la absorción de agua de los elementos de construcción:

- Tasa inicial de absorción (1 minuto);

- Absorción total de agua (24 horas) y

- Absorción en función del tiempo con la determinación de la sorptividad (7 horas).

También fueron caracterizados en relación a las propiedades físicas: porosidad abierta y masa específica aparente. Esta caracterización fue obtenida por la saturación de agua en vacío y siguiendo el procedimiento de Saffiuddin \& Hearn (12). Fueron evaluados 270 bloques de cada serie, con el objetivo de analizar los resultados y conseguir obtener datos que no difieran del 15\% del valor medio determinado en los ensayos de absorción.

Todos los bloques fueron enumerados, pesados y secos durante 24 horas a una temperatura de $110^{\circ} \mathrm{C}$, después fueron enfriados por 24 horas en cajas de poli espuma con sílice gel para evitar la absorción de 
la humedad del medio ambiente. Los bloques fueron envueltos con plástico parafinado hasta la realización de los ensayos y nuevamente se pesaron. Para la realización de los ensayos de tasa inicial de absorción (IRA) y la absorción a través del tiempo fueron considerados los criterios de la norma ASTM C67-11 (13).

Para la realización de los ensayos se construyeron 4 recipientes de acrílico divididos en dos sub-cajas que permitió evaluar 8 bloques por día, como se puede observar en la Figura 1. Para obtener la sorptividad se siguieron los parámetros de la norma ASTM C1585 - 11 (14). La cantidad de agua absorbida en los ensayos de la tasa inicial de absorción y de la absorción en función del tiempo fue determinada por la medición del aumento de la masa, registrados en los intervalos de tiempo: 1 (IRA), 5, 10, 15, 20, 25, 30, 35, 50, 65, 80, 95, 110, $135,180,240,300,360$ y 420 minutos y donde se observó la tendencia de estabilización después de realizados los estudios pilotos preliminares.

\section{RESULTADOS Y DISCUSIONES}

A partir de los resultados de los ensayos de caracterización inicial se verificó que los bloques de hormigón $\mathrm{D}_{1}$ tuvieron una mayor resistencia a la compresión $(7,0 \mathrm{MPa})$ y una absorción total menor $(6,6 \%)$, en relación a los bloques de hormigón $\mathrm{D}_{2}$ que tuvieron una resistencia a la compresión de 4,9 MPa y una absorción total de 7\%. De acuerdo con la norma ABNT 6136 (15), los bloques fueron clasificados como: clase $A E$, en el caso de los bloques $D_{1}$ que permite un uso general para pared externa por encima o por debajo del nivel del suelo, que pueden estar expuesto a la humedad y sin revestimiento de mortero de cemento y clase $\mathrm{BE}$, en el caso de los bloques $\mathrm{D}_{2}$ que permite su uso para un fin específico limitado al nivel del suelo.

Realizada la caracterización de todos los bloques de cada serie (270 bloques) en relación a la tasa inicial de absorción fueron divididos en tres grandes grupos con valores definidos como menores, medios y mayores. La Tabla 1 muestra los resultados para estos grupos.

Seguidamente, se caracterizaron en relación a sus propiedades físicas: masa específica aparente y porosidad abierta, con el fin de comprender la relación entre las características micro-estructurales y las propiedades hidro-físicas. Los valores medios de cada uno de los grupos y para cada una de las series mencionadas con anterioridad se muestran en la Tabla 2.
Como se observa, las dos series de bloques mostraron una variabilidad en relación a las propiedades físicas y microestructurales (16). En el caso de los bloques de la serie $\mathrm{D}_{1}$, presentan coeficientes de variación en el rango del $44 \%$, contrariamente a lo observado en los grupos de la serie $\mathrm{D}_{2}$, donde hay una homogeneidad en los resultados. Se aprecia que los bloques $D_{1}$ presentan una masa específica y porosidad abierta menor en relación a los de la serie $\mathrm{D}_{2}$, verificándose una disminución brusca de las propiedades del grupo 2 para el grupo 3 .

Los bloques fueron subdivididos en 5 subgrupos de 18 bloques y organizados igualmente para cada serie en la siguiente secuencia:

- Grupo 1 - valores menores - Subgrupos V, $\mathrm{VI}, \mathrm{XI}, \mathrm{XII}, \mathrm{XIII}$.

- Grupo 2 - valores medios - Subgrupos VII, VIII, IX, X, XIV.

- Grupo 3 - valores mayores - Subgrupos I, II, III, IV, XV.

A continuación, en la Tabla 3 se presentan los resultados medios de la tasa inicial de absorción, la desviación y el coeficiente de variación para cada subgrupo.

Para los bloques de la serie $D_{1}$, se encontraron valores desde $5,4 \mathrm{~g} / \mathrm{cm}^{2} / \mathrm{min}$ hasta valores de $43,7 \mathrm{~g} / \mathrm{cm}^{2} / \mathrm{min}$. En el caso de los bloques de la serie $D_{2}$, los valores variaron entre $29,8 \mathrm{~g} / \mathrm{cm}^{2} / \mathrm{min}$ a $69,7 \mathrm{~g} / \mathrm{cm}^{2} / \mathrm{min}$. Como se puede observar, los resultados obtenidos corroboran las investigaciones antecesoras en relación a la variabilidad de la tasa inicial de absorción (17). Este ensayo de la tasa inicial de absorción se presenta como una caracterización arbitraria e inconsistente, que no permite una evaluación precisa del comportamiento de los elementos de construcción, por lo que se realizó la determinación de la sorptividad de los bloques.

Tabla 1. Valores medios de la tasa inicial de absorción (IRA).

\begin{tabular}{|c|c|c|c|c|c|c|}
\hline \multirow{2}{*}{ Bloques } & \multicolumn{3}{|c|}{ Serie d1 } & \multicolumn{3}{c|}{ Serie d2 } \\
\cline { 2 - 7 } & Grupo 1 & Grupo 2 & Grupo 3 & Grupo 1 & Grupo 2 & Grupo 3 \\
\hline IRA (g/cm²/min) & 10,49 & 22,31 & 34,63 & 39,44 & 50,75 & 63,81 \\
\hline Desvío & 3,47 & 6,87 & 6,60 & 7,78 & 5,34 & 4,46 \\
\hline Coef_Variación (\%) & 33,04 & 30,79 & 19,06 & 19,74 & 10,51 & 6,99 \\
\hline
\end{tabular}

Tabla 2. Propiedades físicas de los bloques.

\begin{tabular}{|c|c|c|c|c|}
\hline \multirow{2}{*}{ Grupo } & \multicolumn{2}{|c|}{ Porosidad Abierta $(\%)$} & \multicolumn{2}{c|}{ Masa Específica $\left(\mathbf{k g} / \mathbf{d m}^{3}\right)$} \\
\cline { 2 - 5 } & $\mathbf{D}_{1}$ & $\mathbf{D}_{2}$ & $\mathbf{D}_{1}$ & $\mathbf{D}_{2}$ \\
\hline 1 & 17,89 & 21,73 & 2,17 & 2,12 \\
\hline 2 & 16,21 & 18,97 & 2,11 & 2,14 \\
\hline 3 & 6,80 & 16,71 & 0,93 & 2,08 \\
\hline Media & 13,6 & 19,1 & 1,7 & 2,1 \\
\hline Desviación & 6,0 & 2,5 & 0,7 & 0,0 \\
\hline CV $(\%)$ & 43,8 & 13,1 & 40,2 & 1,4 \\
\hline
\end{tabular}

$* \mathrm{~N}=90$ bloques

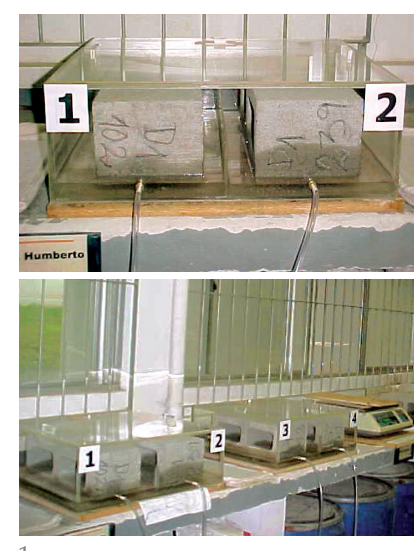

1. Esquema utilizado para la realización del ensayo de IRA y la absorción de agua en un intervalo de tiempo. 
Tabla 3. Valores medios de la tasa inicial de absorción (IRA).

\begin{tabular}{|l|c|c|c|c|c|c|c|c|c|c|c|c|c|c|c|c|}
\hline \multicolumn{10}{|c|}{ Bloques de hormigón_SERIE D1 } \\
\hline Sub-Grupos & I & II & III & IV & V & VI & VII & VIII & IX & X & XI & XII & XIII & XIV & XV & Media \\
\hline IRA (g/cm $/$ /min) & 28,7 & 33,5 & 38,8 & 43,7 & 7,4 & 9,8 & 16,2 & 18,8 & 21,2 & 32,4 & 13,3 & 14,7 & 5,4 & 22,9 & 25,5 & 22,16 \\
\hline Desviación & 0,98 & 1,67 & 1,70 & 1,81 & 0,76 & 1,26 & 0,63 & 0,85 & 0,49 & 7,79 & 0,89 & 0,52 & 0,50 & 1,58 & 0,39 & 1,45 \\
\hline Coef_Variación (\%) & 3,42 & 5,00 & 4,38 & 4,31 & 10,24 & 12,90 & 3,87 & 4,53 & 2,31 & 20,02 & 6,68 & 3,53 & 9,23 & 6,87 & 1,55 & 6,59 \\
\hline \multicolumn{10}{|c|}{ Bloques de hormigón_SERIE D2 } \\
\hline Sub-Grupos & I & II & III & IV & V & VI & VII & VIII & IX & X & XI & XII & XIII & XIV & XV & Media \\
\hline IRA (g/cm²/min) & 62,0 & 65,8 & 69,7 & 59,5 & 30,8 & 41,4 & 51,1 & 54,2 & 55,2 & 30,8 & 44,0 & 48,0 & 29,8 & 51,0 & 59,7 & 50,21 \\
\hline Desviación & 1,41 & 1,01 & 4,38 & 1,97 & 4,96 & 1,91 & 1,23 & 0,52 & 0,64 & 4,96 & 2,41 & 0,90 & 2,61 & 0,68 & 0,68 & 2,02 \\
\hline Coef_Variación (\%) & 2,27 & 1,53 & 6,28 & 3,31 & 16,13 & 4,78 & 2,41 & 0,95 & 1,14 & 16,13 & 5,48 & 1,87 & 8,75 & 1,33 & 1,13 & 4,90 \\
\hline
\end{tabular}

*IRA $\left(\mathrm{g} / \mathrm{cm}^{2} / \mathrm{min}\right)$ - valor medio de $\mathrm{N}=18$

Tabla 4. Valores medios de la Sorptividad para cada Subgrupo de cada serie (S).

\begin{tabular}{|c|c|c|c|c|c|c|c|c|c|c|c|c|c|c|c|c|}
\hline \multicolumn{17}{|c|}{ Bloques de hormigón_SERIE D1 } \\
\hline Sub-Grupos & I & II & III & IV & $\mathrm{V}$ & VI & VII & VIII & IX & $\mathrm{X}$ & $\mathrm{XI}$ & XII & XIII & XIV & XV & Media \\
\hline $\mathrm{S}\left(\mathrm{mm} / \mathrm{min}^{1 / 2}\right) \times 10^{-3}$ & 231 & 191 & 189 & 140 & 209 & 225 & 249 & 261 & 268 & 185 & 247 & 240 & 183 & 252 & 265 & 222,32 \\
\hline Coef_Variación (\%) & 24,80 & 20,23 & 30,17 & 21,93 & 31,58 & 0,00 & 0,00 & 14,44 & 18,82 & 33,99 & 8,35 & 13,91 & 9,94 & 16,1 & 20,2 & 17,63 \\
\hline $\mathbf{R}^{2}$ & 0,96 & 0,95 & 0,97 & 0,95 & 0,99 & 0,99 & 0,98 & 0,98 & 0,97 & 0,95 & 0,99 & 0,98 & 0,98 & 0,98 & 0,99 & 0,97 \\
\hline \multicolumn{17}{|c|}{ Bloques de hormigón_SERIE D2 } \\
\hline Sub-Grupos & I & II & III & IV & $\mathrm{V}$ & VI & VII & VIII & IX & $\mathrm{X}$ & $\mathrm{XI}$ & XII & XIII & XIV & $\mathrm{XV}$ & Media \\
\hline $\mathrm{S}\left(\mathrm{mm} / \mathrm{min}^{1 / 2}\right) \times 10^{-3}$ & 57 & 52 & 47 & 63 & 142 & 116 & 81 & 82 & 66 & 142 & 98 & 96 & 157 & 69 & 67 & 88,99 \\
\hline Coef_Variación (\%) & 31,61 & 41,68 & 41,54 & 41,81 & 26,08 & 25,07 & 39,66 & 45,05 & 40,90 & 26,08 & 30,58 & 32,55 & 21,49 & 42,2 & 21,5 & 33,85 \\
\hline $\mathrm{R}^{2}$ & 0,83 & 0,76 & 0,79 & 0,85 & 0,95 & 0,94 & 0,90 & 0,89 & 0,83 & 0,95 & 0,91 & 0,92 & 0,96 & 0,90 & 0,86 & 0,88 \\
\hline
\end{tabular}

Tabla 5. Valores medios de la Sorptividad (S) para cada grupo de cada serie.

\begin{tabular}{|l|c|c|c|c|c|c|}
\hline \multirow{2}{*}{ Bloques } & \multicolumn{3}{|c|}{ Serie d1 } & \multicolumn{3}{c|}{ Serie d2 } \\
\cline { 2 - 7 } & Grupo 1 & Grupo 2 & Grupo 3 & Grupo 1 & Grupo 2 & Grupo 3 \\
\hline S (mm/min $\left.{ }^{\mathbf{1 / 2}}\right) \mathbf{x}^{\mathbf{1 0}} \mathbf{m}^{-3}$ & 220,80 & 242,99 & 203,16 & 121,76 & 87,94 & 57,27 \\
\hline Coef_Variación (\%) & 12,76 & 16,67 & 23,46 & 27,15 & 38,77 & 35,62 \\
\hline $\mathbf{R}^{2}$ & 0,99 & 0,97 & 0,96 & 0,94 & 0,89 & 0,82 \\
\hline
\end{tabular}

$* \mathrm{~N}=90$ bloques
Los bloques $\mathrm{D}_{1}$ presentaron una capacidad de absorción superior a lo esperado, teniendo en cuenta los resultados obtenidos de la porosidad abierta. Por tanto se deduce de estos resultados, que la relación entre la sorptividad (S) y la porosidad abierta, en el caso de los bloques $\mathrm{D}_{1}$ está vinculado con otras propiedades, sugiriendo, una dependencia más compleja entre la sorptividad y la microestructura del material (tamaño, composición de los poros) (18) (19).

Desde la capacidad de absorción medida a través de la sorptividad se verifica que es posible cuantificar la succión capilar. Sin embargo, la norma ASTM C1585 - 11 (14), solamente permite considerar este valor, si el coeficiente de correlación $\left(R^{2}\right)$ es superior de 0,96. Esto significa que si el valor es inferior, la relación no se considera suficientemente lineal y la sorptividad de la muestra no debe ser determinada a partir de estos datos experimentales (20).

En el caso de los bloques de hormigón $\mathrm{D}_{2}$, que presentaron una absorción brusca en los primeros minutos provocando la saturación del material a los 35 minutos de ensayo, trajo como consecuencia que los resultados derivados de los coeficientes de regresión fueran inferiores a los 0,96. Teniendo en cuenta, lo expuesto anteriormente, los resultados indican: un desvío en el proceso de succión, que el flujo continuo de agua durante el proceso de absorción fue violado y que la teoría de la sorptividad no puede aplicarse para esta serie de bloques. 
En este sentido, surgió una nueva propuesta de caracterización que fue la curva media de absorción de agua a través del tiempo (21). A partir de esta curva media de absorción de agua en función del tiempo se observó que bloques con valores iguales de la tasa inicial de absorción y de la sorptividad presentaron curvas de absorción diferentes. Después de verificar este comportamiento, a partir de la curva de absorción de agua de cada bloque, se reorganizaron y agruparon desde una curva media, que no difiere del $15 \%$ del valor medio de la absorción obtenida en los ensayos individuales y se muestra en las Figuras 2 y 3

Una vez realizado el reagrupamiento de los subgrupos, se obtuvo la curva media de volumen acumulado de agua por unidad de superficie en función de la raíz del tiempo de cada una de las series (270 bloques), que permite apreciar las diferencias y establecer una comparación entre los dos tipos de bloques. (Ver Figura 4)

Como se observa, los bloques de hormigón $\mathrm{D}_{1}$ tuvieron un comportamiento más uniforme en el transcurso del tiempo, dando como resultado valores menores de la tasa inicial de absorción, de la porosidad abierta y de la masa especifica aparente: $22,48 \mathrm{~g} / \mathrm{cm}^{2} / \mathrm{min}, 13,63 \%$ y $1,74 \mathrm{~kg} / \mathrm{dm}^{3}$ respectivamente, pero con valor superior en relación a la sorptividad de 222,05 (mm/ $\min 1 / 2)^{*} 10-3$.

Los bloques de la serie $\mathrm{D}_{2}$, mostraron un comportamiento inverso donde se observa una absorción excesiva de agua en el instante inicial, con una tendencia de estabilización rápida. En los primeros minutos Ilega a la saturación, obteniéndose una menor sorptividad (88.99 (mm/min1/2)*10-3) sin embargo, el coeficientes de correlación no permite tener en cuenta este valor.

Se puede afirmar que las curvas de absorción de agua de los bloques mostraron dos comportamientos: con absorción de agua inicial, caracterizada por una pendiente pronunciada, que de repente se desarroIla hacia la estabilización en el caso de los bloques $\mathrm{D}_{2}$ mientras que los bloques $D_{1}$ tuvieron una absorción inicial menos brusca y con una pendiente más gradual. Estos dos comportamientos también fueron verificadas por Gummerson et al. (22) e Hoffmann (23).

\section{CONCLUSIÓN}

Con esta investigación se constató, que existe una variabilidad en los resultados de la tasa inicial de absorción que confirma las investigaciones antecesoras sobre el asunto.

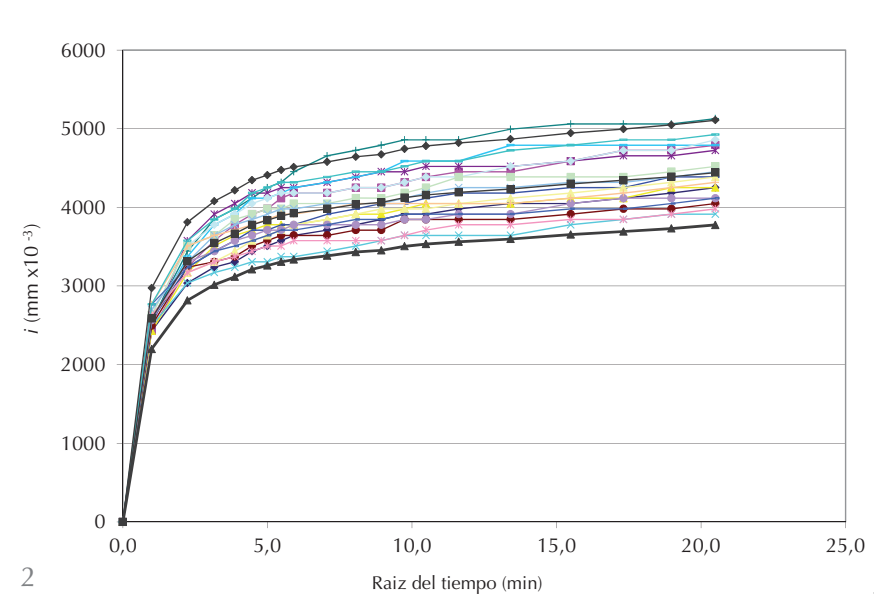

$\rightarrow-221$

$-283$

$\pm 198$

$*-42$

$*-121$

$\bullet-138$

$+246$

$-270$

$-203$

$-267$

$+248$

$*-274$

$\underset{-132}{*-129}$

$-133$

$-135$

$-202$

Superior $15 \%$

- MÉDIA $(38,799$

-Inferior $15 \%$
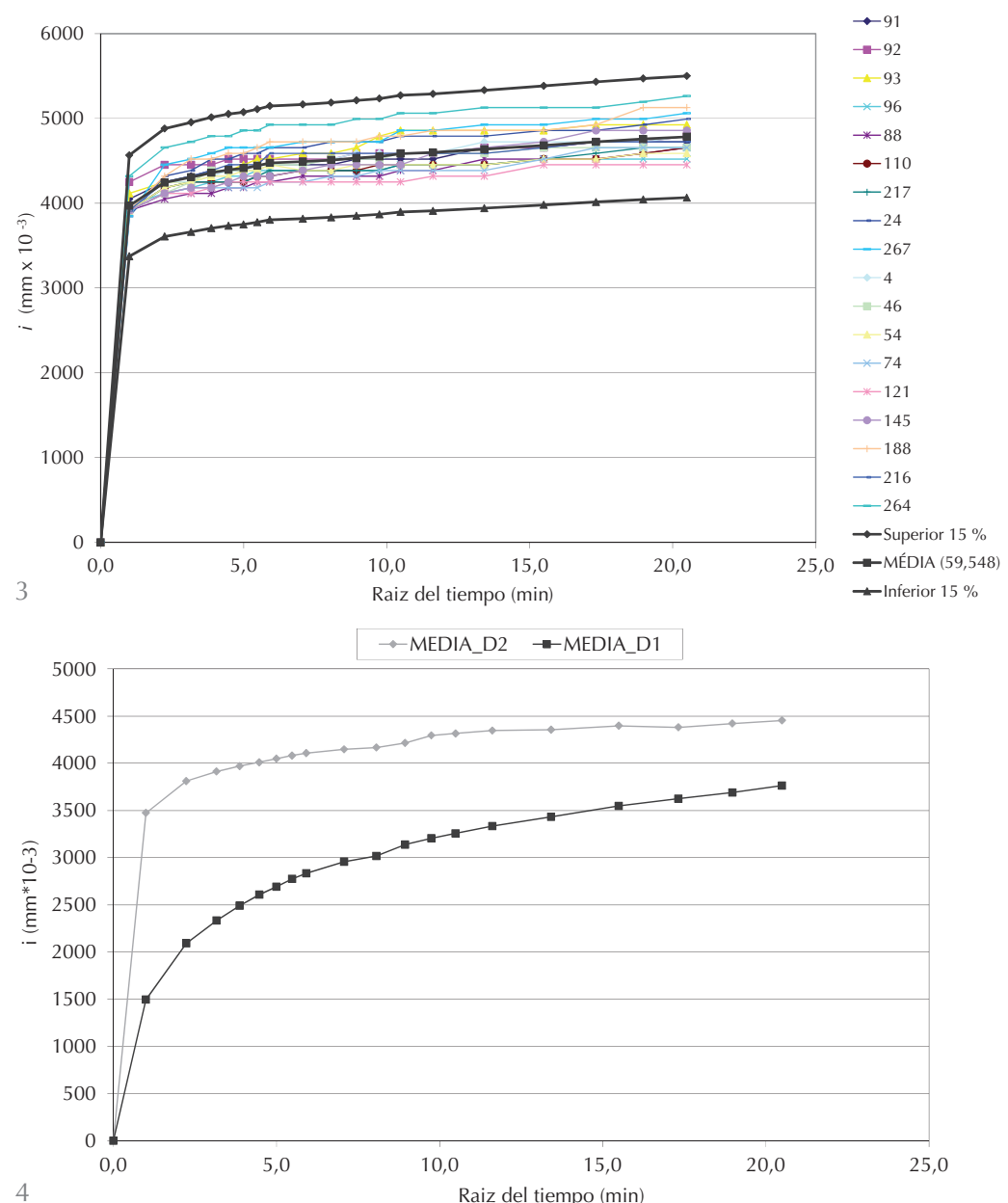

La teoría de la sorptividad en elementos de construcción se ha introducido como un nuevo código de aplicación y criterio de evaluación del desempeño durante la absorción de agua. En este programa experimental se aplicó este código para bloques de hormigón. Los bloques $\mathrm{D}_{1}$ presentaron una sorptividad superior a lo esperado, teniendo en cuenta los resultados obtenidos de la porosidad abierta, corroborando la necesidad de un estudio de la microestructura del material. En el caso de los bloques $\mathrm{D}_{2}$, se observó una alta absorción inicial que llevó a la rápida saturación y a valores menores en relación a la sorptividad, pero
2. Curvas media de absorción de agua en función de la raíz del tiempo para bloques del subgrupo III_D.

3. Curvas media de absorción de agua en función de la raíz del tiempo para los bloques del subgrupo IV_D $\mathrm{D}_{2}$.

4. Curva de comportamiento de la absorción de los bloques de hormigón. 
que estos valores no pueden ser considerados, porque el flujo continuo de agua durante el proceso de absorción fue violado, y la teoría de la sorptividad no puede ser validada.

La curva media de absorción a través del tiempo se presentó como nueva propuesta para la evaluación de la succión capilar, la cual permite verificar el comportamiento de los elementos de construcción desde el instante inicial hasta su saturación, mostrando las particularidades para cada tipo de bloques de hormigón.

\section{AGRADECIMIENTOS}

Los autores agradecen especialmente el apoyo financiero de FINEP y al Consejo Nacional de Investigaciones (CNPq) por la beca otorgada para la realización de esta investigación.

\section{REFERENCIAS}

(1) Audenaert, K., Boel, V., De Shutter, G. (2007). Modelling of capillary suction of water in self compacting concrete. 5th International Essen Workshop. Essen, Germany.

(2) Fishburn, C. C., Watstein, D., Parsons, D. E. (1942). Water Permeability of Walls Built of Masonry Units. BMS_82. National Bureau of Standards. Washington, D. C.

(3) Ritchie, T., Davison, J. I. (1963). Factors Affecting Bond Strength and Resistance to Moisture Penetration of Brick Masonry. Symposium on Masonry Testing. ASTM STP 320, pp. 16-30.

(4) Gallegos, H. (1995). Adhesión entre mortero y las unidades de albañilería. 1. Simpósio Brasileiro de Tecnologia das Argamassas. pp.117-133. Goiânia.

(5) Reda Taha, M. M., El-Dieb, A. S., Shrive, N. G. (2001). Sorptivity: A Surface Absorption Criterion for Brick Units: A Proposal for the Canadian Masonry Standard. 9th Canadian Masonry Symposium. Canadá. Disponible en:www.reda-taha.com/Publications/Fredericton-1-2001.PDF.

(6) Anand, K. B., Vasudevan, V., Ramamurthy, K. (2003). Water permeability of alternative masonry systems. Building Environment, 38(7): 947-957.

(7) Wilson, M.A., Hoff, W.D., Hall, C. (1995). Water movement in porous building materials-XIII. Absorption into a two layer composite. Building Environment, 30(2): 209-219.

(8) Wilson, M. A., Hoff, W. D., Hall, C. (1995). Water movement in porous building materialsXIV. Absorption into a two layer composite (SA < SB). Building Environment, 30(2): 221-227.

(9) Reda Taha, M. M., El-Dieb, A. S., Shrive, N. G. (2001). Sorptivity: a reliable measurement for surface absorption of masonry brick units. Materials and Structures, 34(7): 438-445.

(10) Hanzic, L., Ilic, R. (2003). Relationship between liquid sorptivity and capillarity in concrete, Cement and Concrete Research, 33(9): 1385-1388.

(11) Andrade, L. B., Rocha, J. C., Cheriaf, M. (2007). Aspects of moisture kinetics of coal bottom ash in concrete. Cement and Concrete Research, 37(2): 231-241.

(12) Safiuddin, M. D., Hearn, N. (2005). Comparison of ASTM Saturation Techniques for Measuring the Permeable Porosity of Concrete. Cement and Concrete Research, 35(5): 1008-1013.

(13) American Society for Testing and Materials ASTM C1585-11, 2011. Standard Test Method for Measurement of Initial Rate of Absorption of Hydraulic Cement Mortars and Concretes. 2011.

(14) American Society for Testing and Materials ASTM C 67-11, 2011, Standard Methods of Sampling and Testing Brick and Structural Clay Tile, 2011.

(15) Associação Brasileira de Normas Técnicas. NBR 6136: Bloco vazado de concreto simples para alvenaria estrutural-Determinação da absorção de água, teor de umidade e da área liquida. 1992.

(16) Raimondo, M., Dondi, M., Gardini, D., Guarini, G., Mazzanti F. (2009). Predicting the initial rate of water absorption in clay bricks. Construction and Building Materials, 23(7): 2623-2630.

(17) Hall, M., Djerbib, Y. (2004). Moisture ingress in rammed earth: part 1- the effect of soil particle-size distribution on the rate of capillary suction. Construction and Building Materials, 18(4): 269-280.

(18) Mustelier, N. L., Rocha, J. C., Cheriaf, M. (2011). Estimate of the durability of masonry walls for moisture absorption properties of material constituents. 12DBMC: International Conference On Durability of Building Materials and Components 2011. Porto, Portugal.

(19) Wilson, M. A., Carter, M. A., Hoff, W. D. (1999). British Standard and RILEM water absorption tests: A critical evaluation. Materials and Structures, 32(8): 571-578.

(20) Reda Taha, M. M., El-Dieb, A. S., Shrive, N. G. (2001). The Theory of Sorptivity: Application to Masonry Brick Units. 9th International Colloquium on Structural and Geo-technical Engineering. Cairo, Egypt.

(21) Paes, I. N. L. (2004). Avaliação do transporte de água em revestimentos de argamassas nos momentos iniciais de pós-aplicação (Tese de Doutorado em Estruturas e Construção Civil). Universidade de Brasília. (en portugués).

(22) Gummerson, R. J., Hall, C., Hoff, W. D. (1981). The suction rate and the sorptivity of bricks. Transactions and Journal of the British Ceramic Society, Vol. 80, pp. 150-152.

(23) Hoffmann, D., Niesel, K. (1988). Quantifying capillary rise in columns of porous material. American Ceramic Society Bulletin, 67(8): 1418. 